\title{
On contact numbers in random rod packings
}

\author{
A. Wouterse - S. Luding • A. P. Philipse
}

Received: 15 November 2007 / Published online: 5 February 2009

(C) The Author(s) 2009. This article is published with open access at Springerlink.com

\begin{abstract}
Random packings of non-spherical granular particles are simulated by combining mechanical contraction and molecular dynamics, to determine contact numbers as a function of density. Particle shapes are varied from spheres to thin rods. The observed contact numbers (and packing densities) agree well with experiments on granular packings. Contact numbers are also compared to caging numbers calculated for sphero-cylinders with arbitrary aspect-ratio. The caging number for rods arrested by uncorrelated point contacts asymptotes towards $\langle\gamma\rangle=9$ at high aspect ratio, strikingly close to the experimental contact number $\langle C\rangle \approx 9.8$ for thin rods. These and other findings confirm that thin-rod packings are dominated by local arrest in the form of truly random neighbor cages. The ideal packing law derived for random rod-rod contacts, supplemented with a calculation for the average contact number, explains both absolute value and aspect-ratio dependence of the packing density of randomly oriented thin rods.
\end{abstract}

Keywords Random packings - Rods - Contact numbers . Spherocylinders $\cdot$ Computer simulations $\cdot$ Molecular dynamics

\footnotetext{
A. Wouterse - A. P. Philipse $(\bowtie)$

Van't Hoff Laboratory for Physical and Colloid Chemistry,

Utrecht University, Padualaan 8, 3584 CH Utrecht,

The Netherlands

e-mail: a.p.philipse@uu.nl

S. Luding

Multi Scale Mechanics, Universiteit Twente, CTW, TS,

P.O. Box 217, 7500 AE Enschede, The Netherlands
}

\section{Introduction}

The random rod packing is a valuable reference for packed granular matter composed of elongated particles as can be found in fiber-reinforced and other fibrous materials [1-4], and anisotropic powders [5,6]. This reference packing is a stacking of randomly oriented, rigid rods with a maximum particle volume-fraction uniquely determined by the rod aspect ratio [5]. Similarly, disorderly packed granular spheres are modeled by the (Bernal) random sphere packing [7]. Spheres and rods actually belong to a whole family of random particle packings, with a density fixed by the particle shape $[5,8]$. Interestingly, the maximum density does not occur for the Bernal sphere packing, but for slightly deformed spheres. This density maximum was first found for sphero-cylinders [8] and later also for prolate spheroids [9] and cut spheres [10].

An essential difference between randomly packed spheres and thin rods is the following. Due to the excluded volume effects there are strong positional correlations in a sphere packing that are very difficult to incorporate in a packing law, i.e. a general relation between packing density, particle shape and contact numbers. Despite additional rotational degrees of freedom, it is much easier to model the random packing density for rods, because correlations between rodrod contacts vanish in the thin-rod limit [5]. This asymptotic behavior has been clearly confirmed by simulations [8]. Absence of contact correlations entails an "ideal" packing law (see [5] and also Sect. 2.2), namely a linear dependence of the random rod packing density on the average contact number $\langle C\rangle$.

The evaluation of $\langle C\rangle$, however, is not trivial. Philipse [5] concluded from a fit of experimental rod packing densities to the thin-rod packing law that $\langle C\rangle=10.8 \pm 0.4$. Only recently Blouwolff and Fraden [11] succeeded to directly 
count contacts in experimental random rod packings and they report that for thin rods $\langle C\rangle$ is about 10 . The authors rationalized this outcome via an "isostatic" argument to which we return in Sect. 2.2. Experiments on non-spherical particle packing have also been performed by Stokely et al. [12], Desmond and Franklin [13] and Lumay and VandeWalle $[14,15]$.

Computer simulations of random rod packings are needed, not only for better understanding experimental densities and contact numbers, but also to investigate issues that are experimentally difficult to assess. (An example is the contact number as function of particle volume fraction, treated in Sect. 3.1). For random packing of non-spheres simulation algorithms are available, such as molecular dynamics [16], discrete element method [17], event-driven molecular dynamics [18,19] and the mechanical contraction method (MCM). The MCM generates reproducible random packings of spheres and various non-spherical shapes $[8,10]$ with densities that are slightly below values from event driven molecular dynamics [9]. However, the MCM has yielded contact numbers that are unphysical for high aspect ratio spherocylinders. Defining a contact via a threshold distance [8] yielded for thin spherocylinders that $\langle C\rangle$ is about 3, which is too low to achieve mechanical stability and anyhow much below the experimental values mentioned above.

The aim of this study is firstly to reproduce experimental contact numbers by combining MCM and molecular dynamics, secondly to investigate the dependence of $\langle C\rangle$ on the particle volume fraction and finally, to analyze contact numbers in terms of a mathematical caging problem. In Sect. 2.1 we describe the simulation method and the evaluation of contact numbers employing expansion of particles that interact via a spring-dashpot model. The caging problem, i.e., finding the average minimal number of uncorrelated contacts needed to arrest a particle, has only been solved for spheres [20,21] and 2-dimensional discs [22]. In Sect. 2.2 we explain a numerical solution of the caging problem for sphero-cylinders with arbitrary aspect-ratio. One of the issues in the discussion in Sect. 3 is whether magnitude and aspect-ratio dependence of contact numbers for randomly packed rods can be explained as a local caging effect.

\section{Methods}

\subsection{Simulation method}

The starting configuration is a random particle packing generated with the MCM [8]. The particles are then expanded in steps at a constant growth rate. Contacts created between particles are modeled as spring-dashpots following Silbert et al. [23] for spheres and Pournin et al. [24] for spherocylinders. The approach of these authors was adopted in the

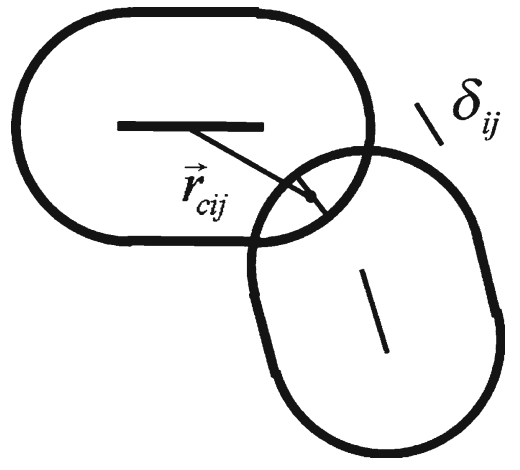

Fig. 1 Illustration of the overlap $\delta_{i j}$ that is shown as a line segment, $\hat{n}$ is the unit vector that points into the direction of the line segment and $\mathbf{r}_{c i j}$ is the branch vector connecting the center of mass of a particle with the center of the overlap line, i.e. the contact

sense that only the normal component of the overlap was considered whose magnitude is calculated as the diameter minus the shortest distance between the two line segments forming the spherocylinders (see Fig. 1). The contact force acting between overlapping particles is then given by

$\mathbf{f}=-\left(k \delta+\gamma \mathbf{v}_{r e l} \cdot \hat{n}\right) \hat{n}$

where $k$ is a spring constant, $\delta$ is the amount of overlap between two particles, $\gamma$ is a viscosity constant, $\mathbf{v}_{r e l}$ is the relative velocity of the two particles and $\hat{n}$ is a unit vector in the direction of the shortest distance between the two line segments forming the two spherocylinders. All particles experience a small background viscosity force but friction between particles is not modeled. Typical simulation parameters are $k=10^{5}, \gamma=10, \gamma_{b g}=0.1$ and $d t=10^{-5}$ during the growth and $10^{-4}$ during relaxation. The mass of a particle is equal to the particle volume, i.e. density is equal to one. The particle positions are updated by integrating the Newton-Euler equations of motion using a simple Euler scheme [25]:

$$
\begin{aligned}
\dot{x} & =v \\
\dot{p} & =f \\
\dot{q} & =\frac{1}{2} \omega q \\
\dot{L} & =\tau
\end{aligned}
$$

where $q$ is a quaternion representing the orientation of a particle, $\omega$ is the angular velocity, $L$ is the angular momentum and $\tau$ is the total external torque.

The expansion of particles is terminated when a user -specified volume fraction is reached and after that the system of spheres is allowed to relax to zero total (kinetic + potential) energy within the numerical error. Then the size of the particles is increased again by a small increment such that the volume fractions increases by 0.1 and the relaxation is repeated. The final volume fraction is reached when it is 
no longer possible for the system to relax to zero potential energy by reorganization in response to the overlaps (forces) created. The expansion rate and viscosity of the particles were chosen such that the kinetic energy component of the total energy is about 10-100 times smaller than the potential energy on average during a simulation run in order to keep the final structure as close as possible to the original starting configuration generated with the MCM and to maintain a disordered structure during the simulation. The final kinetic energy of the spheres is zero within the numerical error. The method described here is similar to [26,27], where disordered collections are created by minimizing potential energy using a conjugate gradient method. In these studies particles can grow or shrink depending on whether the configuration is above or below the jamming point.

\subsection{Caging of non-spherical particles}

The random contact equation [5] states that for a random packing with completely uncorrelated contacts, the volume fraction $\phi$ is given by

$\phi=\frac{\langle C\rangle V_{p}}{V_{\text {excl }}}$

where $\langle C\rangle$ is the average contact number per particle, $V_{p}$ the particle volume and $V_{\text {excl }}$ the orientationally averaged excluded volume for two particles, i.e., the volume that cannot be occupied by the center of mass of one spherocylinder with fixed orientation when sliding over another spherocylinder. For spherocylinders with diameter $D$ and a length $L$, the excluded volume [28] is

$V_{\mathrm{excl}}=\frac{4}{3} \pi D^{3}+2 \pi L D^{2}+\frac{\pi}{2} D L^{2}$

Substituting (4) into (3) we find for the limit of thin rods:

$\phi \frac{L}{D} \approx \frac{\langle C\rangle}{2} ;$ for $\frac{L}{D} \gg 1$

This packing law for random thin rods has been verified in experiments [5,11] as well as simulations [8].

To calculate a value for $\langle C\rangle$ we note that in a rod packing almost all particles are caged/jammed i.e. the particles cannot move because their movement is blocked by neighboring particles. Consequently, an appropriate mathematical approximation for $\langle C\rangle$ is to calculate the caging number $\langle\gamma\rangle$, defined as the minimum average number of contacts required to immobilize a particle by randomly placed contacts, where a contact can be a fixed point or another particle. Analytical solutions exist for 2-dimensional disks [21] and for 3 -dimensional spheres caged by point contacts [20]. In [22] a caging number is calculated for rods $(\langle\gamma\rangle=5)$ where only the translations are blocked, which is a lower bound for the contact number in random rod packings.
Here we show how to calculate the caging number $\langle\gamma\rangle$ for general shapes using a linear algorithm. In [29] it is shown that the relative acceleration of $n$ contact points under the application of non-zero forces in a collection of rigid bodies can be written as the linear equation

$A \mathbf{f}+\mathbf{b}=\mathbf{a}$

where $A$ is a symmetric and positive semi-definite $n \times n$ matrix, $\mathbf{f}$ is a vector with all elements larger than or equal to zero, $\mathbf{f} \geq 0$, representing a positive pushing force, $b$ is a vector that takes external forces into account and $\mathbf{a}$ is a vector whose elements consist of the relative accelerations of the $n$ contact points. Rigid bodies are not allowed to overlap, which requires $\mathbf{a} \geq 0$, which can be written as a so-called Linear Complementarity Problem (LCP) [30,31], which is defined as follows:

$A \mathbf{f}+\mathbf{b} \geq 0, \quad \mathbf{f} \geq 0 \quad$ and $\quad \mathbf{f}^{T}(A \mathbf{f}+\mathbf{b})=0$

In the caging problem contacts are randomly placed on a rigid body until all translations and rotations are blocked for that body. A body is caged if the relative acceleration at all contact points is zero under the application of a non-zero force [32] and we can use a LCP solver to determine whether a body is caged or not by examining the total force acting on a body. For a spherocylinder, fixed contact points are randomly distributed on the spherocylinder with the restriction that the probability to place a contact on a hemi-spherical cap or the cylindrical part is proportional to their respective surface areas.

\section{Results and discussion}

\subsection{MCM+MD hybrid simulation results}

A random sphere gas consisting of 2,048 particles is contracted with the MCM to a volume fraction of 0.49 . The spheres are then grown in size at various growth rates and the average contact number was recorded as a function of volume fraction (Fig. 2a). Note that only the final points in Fig. 2a, b corresponds to mechanically stable packings, whereas the other points represent intermediate states during the simulation. A lower growth rate keeps the spheres in contact resulting in a higher contact number. At a volume fraction of around 0.60 the average contact number becomes the same irrespectively of the growth rate. Similar results were obtained in the work of Silbert et al. [23] where the packing properties depended on the coefficient of restitution and the friction coefficient but became independent of simulation parameters in the limit of close packing. The same procedure was followed for spherocylinders of aspect ratio 1.5, 6 and 11 with results shown in Fig. 2b. Note that the generated packings are not stable at low volume fractions 
Fig. 2 Evolution of contact number as a function of volume fraction for random packings with expanding particles. a Spheres with different growth rates; b Spherocylinders with aspect ratio $\alpha=\frac{L}{D}=1.5$ (circles), 6 (triangles) and 11 (squares)

Fig. 3 Distribution of contact numbers in random rod packings at different volume fractions for aspect ratio $\alpha=1.5$ (a) and 11 (b). Black bars represent the maximum random packing density for the given aspect ratio (a)

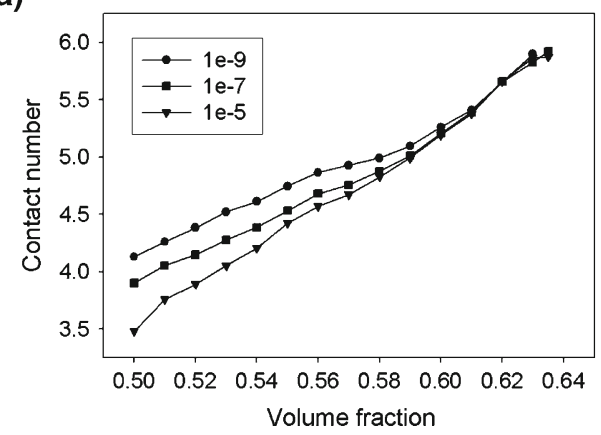

(a)

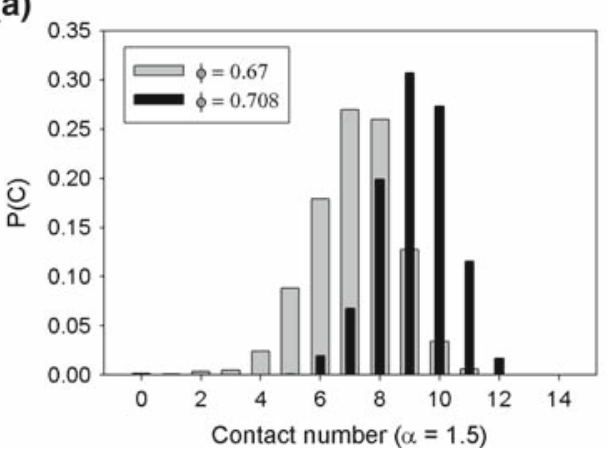

(b)

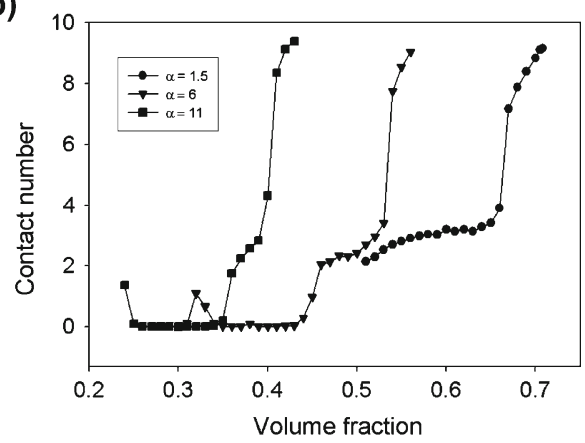

(b)

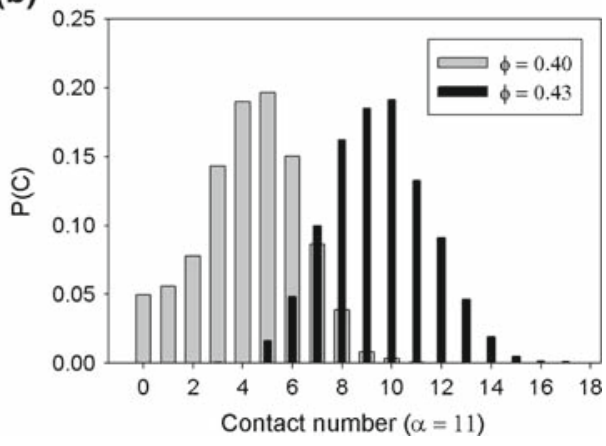

and should be considered as disordered collections of particles in contact. Only above a certain volume fraction (different for different aspect ratios) do the packings become stable — as indicated by the jump in Fig. 2b. For frictionless spheres the strict criterion for stability is $C \geq 6$ (if rattlers are excluded) but we do not exclude rattlers here so that $\mathrm{C}$ for a stable packing can be somewhat smaller than 6 . Note that there is no jump around the stability onset, but the different growth rates converge. In contrast to spheres, where the contact number increases roughly linearly with volume fraction, spherocylinders show a sharp non-linear increase in contact number from about three up to a value between 9 and 10. Reducing the growth rate leads to a less steep increase.

As the volume fraction increases the distribution of contact numbers keeps roughly the same shape (Fig. 3) but the average shifts to a higher value. Note also that the average contact number (Fig. 2b) at the maximum density is fairly insensitive to the aspect ratio. This is consistent with the trend in the average caging number below in Fig. 10.

The sharp non-linear increase in contact number in Fig. 2b for the spherocylinders could indicate a phase transition such that upon increasing the volume fraction of the random spherocylinder packings, the amount of order is increased in the packings due to alignment of spherocylinders. Graphical rendering of the packings (Fig. 4), however, shows that the sharp increase in Fig. 2b is not caused by particle alignment but apparently by particles suddenly coming into contact with each other
A study of the nematic order parameter $S$ (Fig. 5) confirms that the packings remain disordered $(S \approx 0)$ as the particles grow in size, however, the spikes in the order parameter indicate small orientational changes in the packing. Donev et al. [33] calculated $S$ for spheroids and found an experimental value in the order of 0.05 consistent with the values in Fig. 5.

As the volume fraction increases, more particles come into contact and motion is progressively hindered. The percentage of caged particles was calculated, defining a caged particle as a particle whose translations and rotations are blocked by the presence of its contacting neighbors [20,21]. It should be noted that the percentage of caged spheres does not provide a criterion for the stability of a sphere packing. A packing can be static when non-caged spheres rest on other spheres. Furthermore, caging is a local criterium and for modeling global jamming more complicated linear programming algorithms are necessary [34] but it appears that long thin rods form an exception to this due to their highly uncorrelated entanglement. Nevertheless, a packing with a large percentage of non-caged spheres will certainly be unstable.

Slower expansion rates produce packings with more caged spheres as can be seen in Fig. 6a. The starting configuration was generated with the MCM. Possibly, a slow expansion rate leaves the cages intact whereas a higher rate destroys the initial cages, resulting in more non-caged spheres at lower volume fractions. A growth rate lower than $10^{-9}$, for example $10^{-10}$, did not produce more caged spheres but a percentage similar to $10^{-9}$. This explanation is supported by the graph of the contact number versus volume fraction (Fig. 2a): for 
Fig. 4 Graphical rendering of several packings. a Rods with aspect ratio $\alpha=1.5$ at $\phi=0.51$ and at their maximum random packing density, $\mathbf{b} \phi=0.708$. c Rods with aspect ratio 11 and $\phi=0.40$ and at their maximum density, $\mathbf{d} \phi=0.43$. These images indicate that the steep rise in contact number in Fig. $2 b$ is not accompanied by a significant structural change (a)

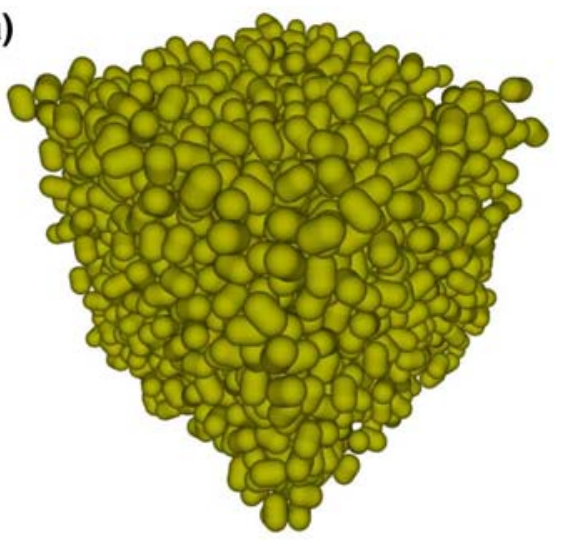

(c)

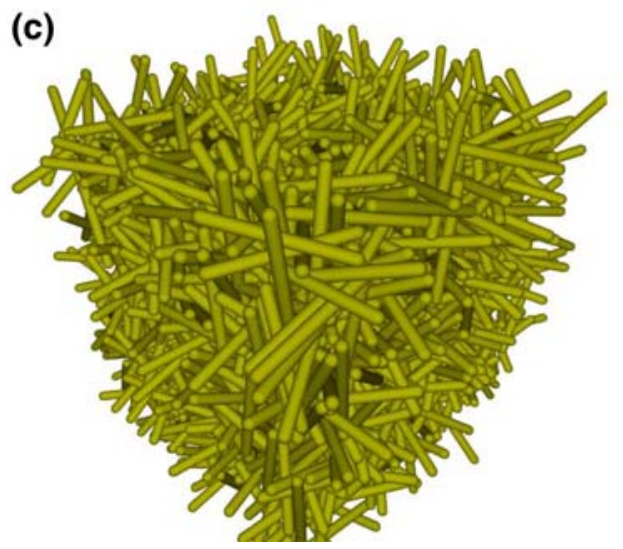

(b)

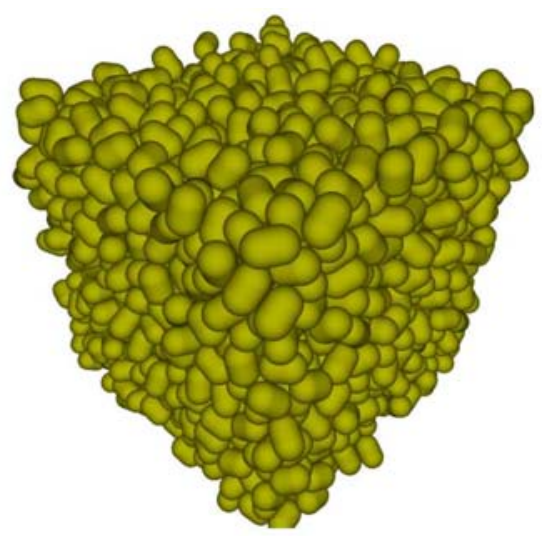

(d)

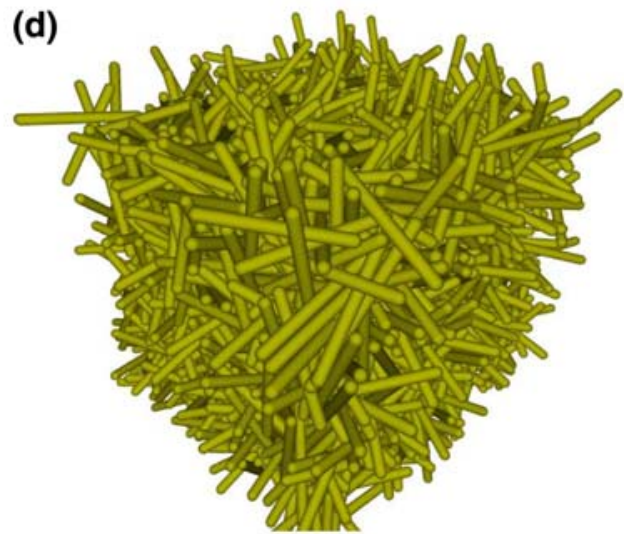

higher growth rates the contact number is lower at the same volume fraction indicating a higher percentage of non-caged spheres. At around 0.61 volume fraction the percentage of non-caged spheres converges for all growth rates while for $\alpha=1.5$ this rate independence is obtained for $\phi>0.68$, see Fig. $6 b$.

The caging of spherocylinders was studied as described in Sect. 3. In Fig. 6b the percentage of non-caged particles is plotted as a function of volume fraction for different growth rates. For the caging of rods only the blocking of translations and rotations perpendicular to the axis of symmetry were checked. The percentage of non-caged spheres and spherocylinders is directly correlated to the contact number as can be seen by comparing Figs. 2 and 6.

Figure 2b, and Fig. $6 c$ also shows clear similarities; when the contact number sharply increases, the fraction of noncaged particles sharply decreases. Plotting the percentage of non-caged particles as a function of contact number (Fig. 7) shows that the percentage of non-caged particles is almost independent of the aspect ratio.

\subsubsection{Aspect ratio dependence for random rod packings}

So far packing properties of spherocylinders with three different aspect ratios have been studied with the hybrid

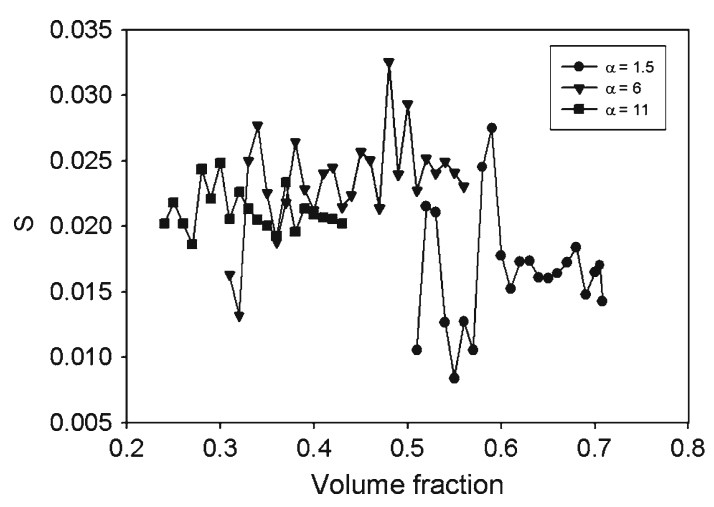

Fig. 5 Order parameter $S$ as a function of volume fraction for spherocylinders with aspect ratio $\alpha=1.5$ (circles), 6 (triangles), 11 (squares). Upon increasing the volume fraction no significant increase in the amount of order is observed

MCM-MD method, which yielded packings with different volume fractions but having roughly the same contact number. To see how the contact number depends on the aspect ratio, and whether it depends on the method, we now consider in more detail the volume fraction and contact number as a function of aspect ratio. In Fig. 8 the volume fraction of a random spherocylinder packing is plotted as a function of aspect ratio for the MCM and the MD simulation of growing particles. The shapes of the curves are very 
Fig. 6 Fraction non-caged particles as a function of volume fraction for different expansion rates; a spheres,

b spherocylinders with aspect ratio $\alpha=1.5$, $\mathbf{c}$ Fraction of non-caged rods with aspect ratio $\alpha=1.5$ (circles), 6 (triangles) and 11 (squares) in the quasi-static limit with growth rate $10^{-7}$ (a)

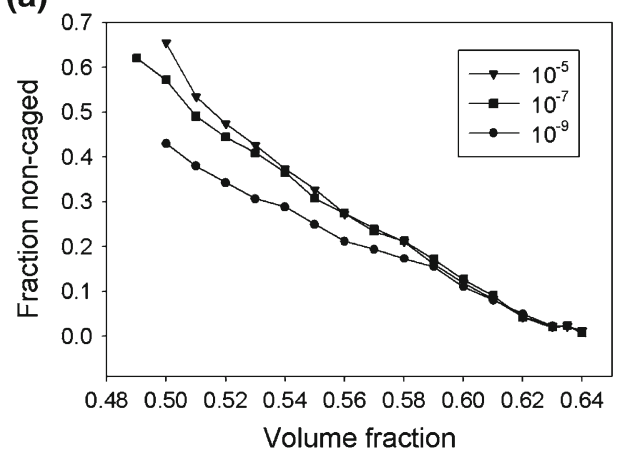

(b)

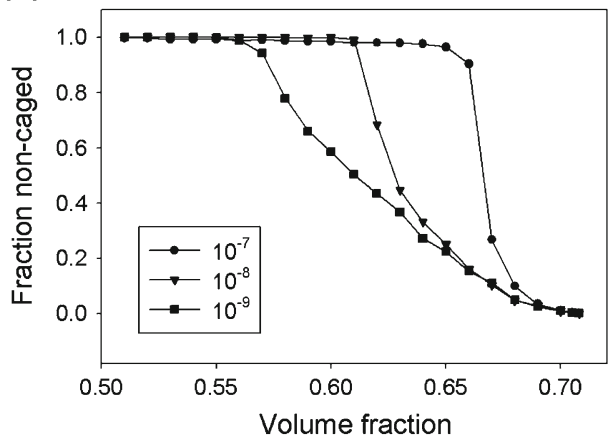

(c)

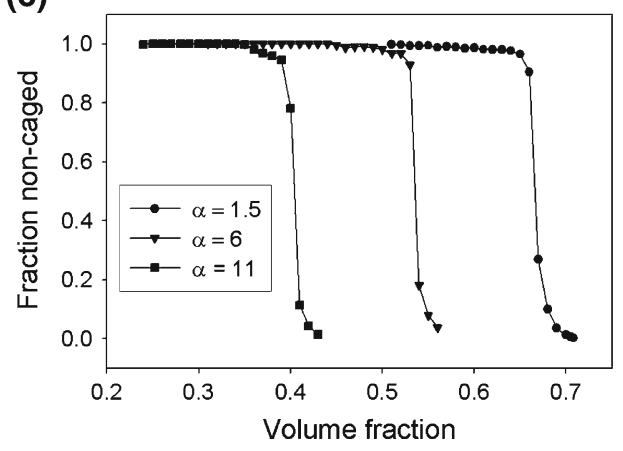

similar and the volume fraction obtained via the MD simulation is slightly higher, as expected, since the MCM packings are used as starting configuration for the growth procedure. Note that the simulation data approach the thin-rod packing law Eq. (5) at an aspect ratio in the range 10-20. This is also the aspect ratio range in Fig. 10 in which the caging number asymptotes to its constant value in accordance with Eq. (5).

Though the volume fractions between the two methods do not differ much, there is a difference in the contact number (Fig. 9). For the MD simulation the contact numbers are clearly higher than for MCM and comparable to the results from Blouwolff and Fraden [11] for uncompacted rods. Blouwolff and Fraden give an argument for the contact number in random rod packings, which we briefly recapitulate here. For $N$ rods, there will be $N_{c}=N\langle C\rangle / 2$ contacts and $N_{c}$ contact equations that must be satisfied. Rods have 5 degrees of freedom, so there are a total of $5 N$ variables specifying the configurations of the rods. The number of constraint equations must be less than the number of variables, yielding $N\langle C\rangle / 2 \leq 5 N$ and $\langle C\rangle \leq 10$. Mechanical stability gives 5 force-torque equations per particle. The number of mechanical stability equations cannot exceed the number of force variables, thus, $5 N \leq N\langle C\rangle / 2$ or $10 \leq\langle C\rangle$. Combining these two limits gives the isostatic value $\langle C\rangle=10$. In $[10,35]$ it is noted that the isostatic contact value is not reached when spherocylinders are only slightly deviating from spheres. The number of degrees of freedom changes discontinuously and via the isostatic conjecture the number of contacts should

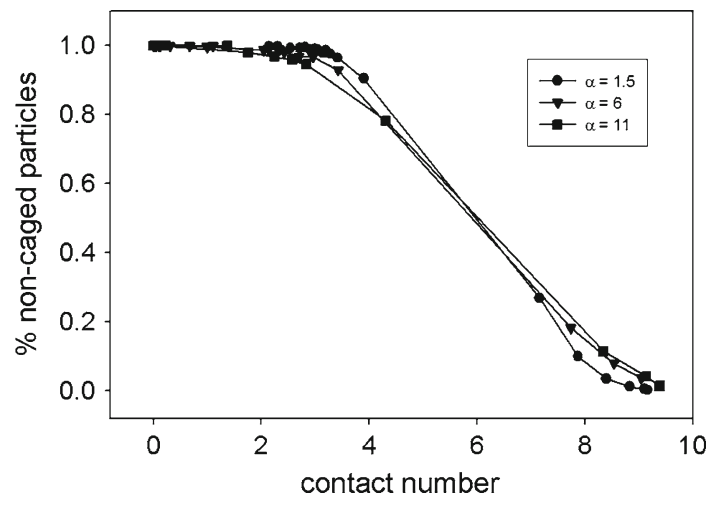

Fig. 7 Fraction of non-caged particles as a function of contact number from data of Fig. 2b, and Fig. 6c showing that the percentage of noncaged particles is almost independent of the aspect ratio for growth rate $10^{-7}$

change discontinuously too which, however, is not observed in computer simulations.

In Fig. 9 the contact number is plotted as a function of aspect ratio for random spherocylinder packings. The contact number increases monotonically and asymptotes towards a value between 9 and 10, which agrees quite well with the direct experimental value $\langle C\rangle=9.8 \pm 0.3$ found in [11]. The finding that $\langle C\rangle$ asymptotes towards a constant value implies according to the random contact equation (1.5) the scaling $\phi L / D=\frac{\langle C\rangle}{2}$ for $L / D \gg 1$, which has also been found experimentally $[5,11]$. In the next section a physical explanation for this constant $\langle C\rangle$ will be given in terms of a local 
Fig. 8 a Volume fraction of a random spherocylinder packing as a function of aspect ratio (circles) MCM and (triangles) MD. The line corresponds to Eq. (5). b Log-log plot of the volume fraction as a function of aspect ratio with slope -1.08
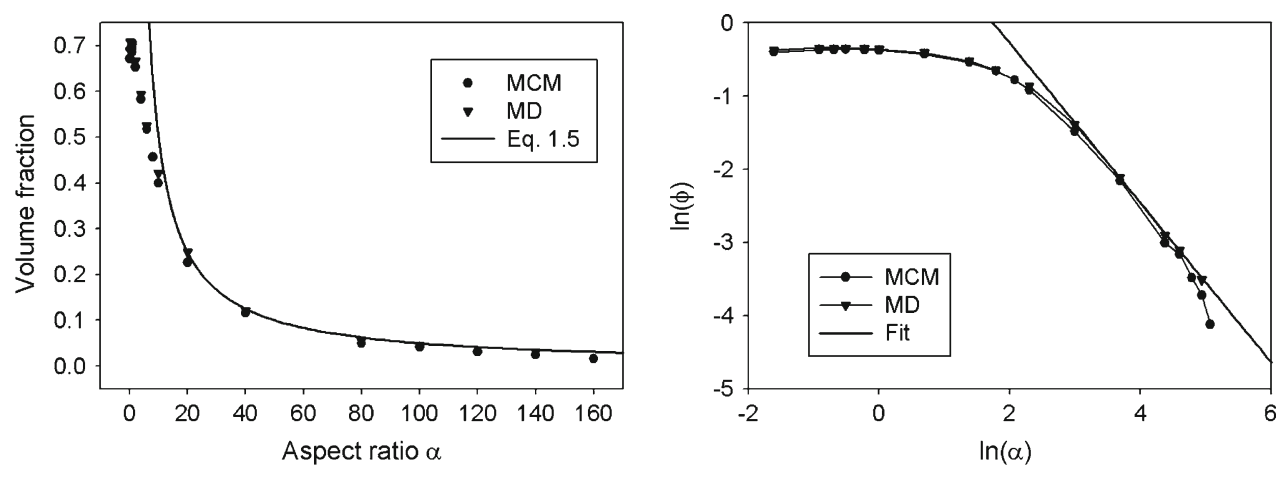

Fig. 9 Contact number $\langle C\rangle$ as a function of aspect ratio for random spherocylinder packings at the close packing volume fraction. The dip in contact number after aspect ratio 6 is probably due to the packings being not completely jammed and further densification should be possible

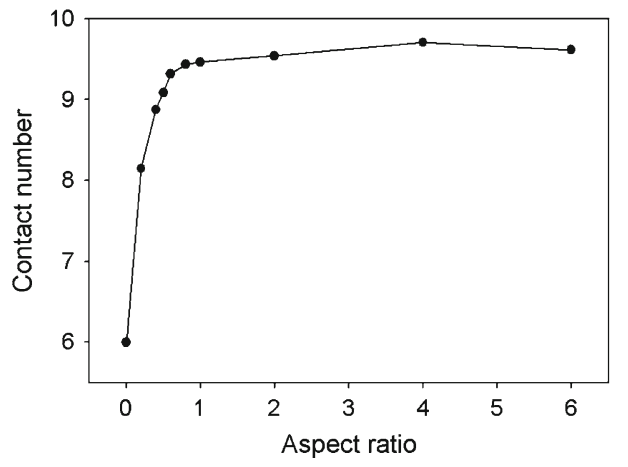

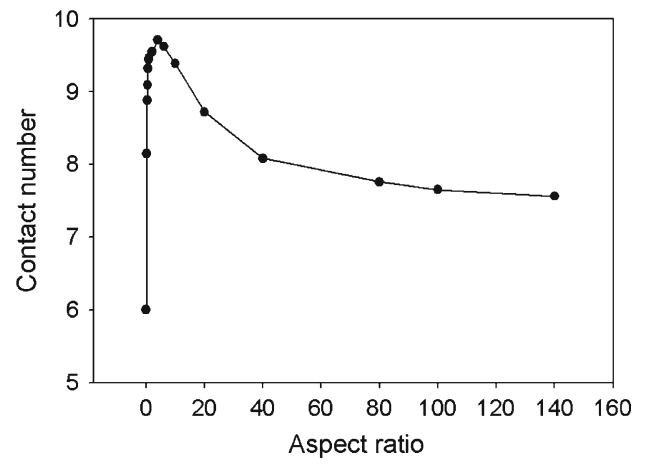

caging argument. The molecular dynamics procedure allows for slightly denser packings with better-defined contacts. The value of 10 is not reached but possibly implementing a "shaking" algorithm, which perturbs the packings without inducing an ordered phase allows for further densification and increase of contact number.

\subsubsection{Contact number as a function of aspect ratio}

In [32] it was shown that the parking number for spheres is about 8.4 but that it is not possible to pack each sphere with that contact number locally due to excluded volume effects. A contact number below the parking number is more optimal for a dense disordered packing of spheres. When slightly deviating from spheres the surface area of the particles hardly changes and also the excluded area as described for spheres in Wouterse et al. [21] will not alter significantly. Thus the parking number [36] for small aspect ratio spheroids and spherocylinders is expected to be around the same value as for spheres, which is below 10 and the parking number is an upper bound for the number of contacts that can be placed at random. It should be noted that the parking number is not really well-defined for higher aspect ratio spheroids and spherocylinders. As contacts are added randomly the available parking space decreases due to the non-overlap condition. At a certain point only spherocylinders with the right orientation will fit in that space which induces alignment in the particles.

\subsection{Caging number for spherocylinders}

Using the formalism described in Sect. 2.2 we reproduced the exact caging number $\langle\gamma\rangle=5$ for two-dimensional disks caged by uncorrelated point contacts [22]. We determined the caging number for infinitely long rods, considering only translations and rotations perpendicular to the axis of symmetry to be blocked (thus the rods can always move along and rotate around their long axis). The result for two- and threedimensional rods is, respectively, $\langle\gamma\rangle=5$ and $\langle\gamma\rangle=9$. The caging number for spherocylinders as a function of aspect ratio was calculated (Fig. 10) where contacts are placed randomly on the cylindrical part or the hemi-spherical end caps with a probability proportional to the surface area of the cylindrical respectively hemi-spherical surface. For aspect ratio zero the caging number for $3-\mathrm{d}$ spheres $\langle\gamma\rangle=7$ is reproduced numerically; a number derived analytically elsewhere [20]. For high aspect ratios $\langle\gamma\rangle$ approaches the value of 9 for infinite rods, as expected, since the probability of placing a contact on an end cap vanishes in the thin rod limit. Interestingly, immediately upon deviating from spheres (see Fig. 10) the caging number jumps to around 12 and then starts to decrease.

Although we have reproduced limiting cases with our method of which we have the analytical solutions, it is difficult to numerically solve the LCP and it is not completely sure whether the results for almost spherical particles are numerically robust. However, the increase in caging number as such 
(a)

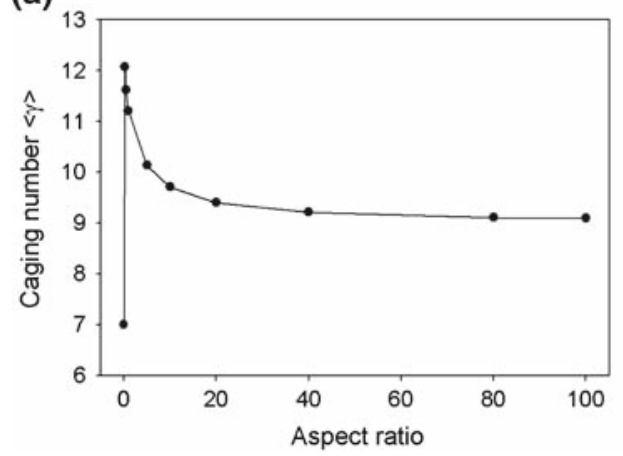

(b)

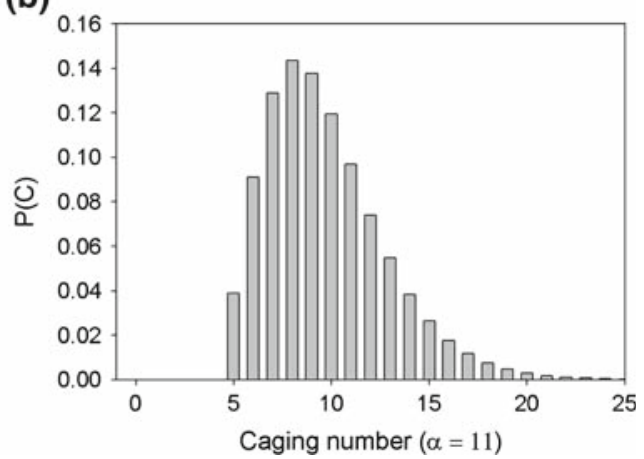

Fig. 10 a Average number of contacts required to block rotations and translations perpendicular to the axis of symmetry for a spherocylinder as a function of its aspect ratio, computed as discussed in Sect. 2.2. b Distribution of contacts for the caging number of a spherocylinder with aspect ratio 11

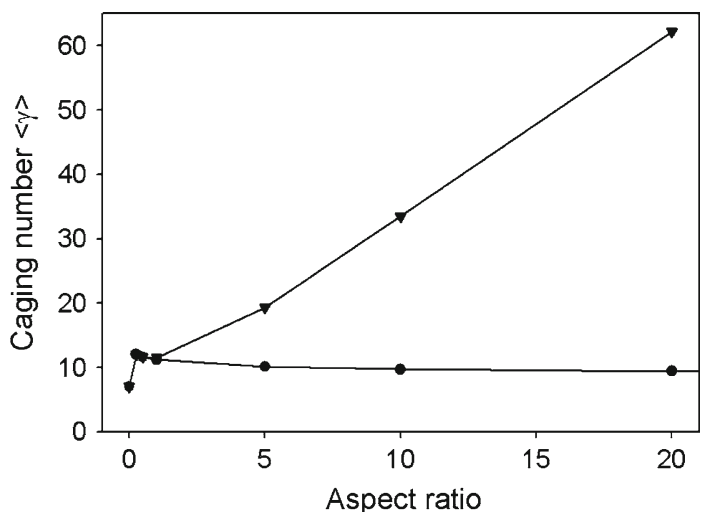

Fig. 11 (triangles) Average number of contacts required to block rotations perpendicular to the axis of symmetry and all translations for a spherocylinders as a function of its aspect ratio (circles; Fig. 10a)

is physically plausible since the volume fraction of a random packing increases upon slightly deviating from spheres which supports the fact that it becomes harder to cage or jam the non-spherical particles.

Even though the caging number is a local contact number, the limiting value of $\langle\gamma\rangle=9$ is surprisingly close to the isostatic value of 10 and average contact numbers from experiments $\langle C\rangle=10.8 \pm 0.4$ [5] and $\langle C\rangle=9.8 \pm 0.3$ [11]. Somewhere in the aspect ratio range 15-20 the caging number has become almost constant which is where the random contact equation yields a good comparison with experiments $[5,8]$. High aspect ratio thin rods have uncorrelated contacts making the point contact approximation a valid choice where the difference in contact number between 9 and 10 could make sense to satisfy global jamming conditions. Interestingly, our results match well with simulations on semi-flexible rods, where a caging number of 8 was found [37].

In Fig. 11 the caging number is plotted as a function of aspect ratio, for the case where also translations along the axis of symmetry are required to be blocked. Here, the caging number is completely dominated by the probability to place a contact on both hemi-spherical caps, which becomes infinitesimally small in the limit of infinite aspect ratio yielding a sharp increase in the contact number. In view of the experimental and simulated contact numbers it is clear that in random thin rod packings the large majority of rods is unblocked at its ends. It should be noted that any small motion of rods parallel to their main axis has little effect on the random packing density because these axes are randomly oriented.

\section{Conclusions}

Performing molecular dynamics simulations on packings prepared with the MCM is an efficient way for generating random packings with volume fractions and contact numbers comparable to experimental packings. In the limit of jamming, the packing properties such as contact number and percentage of caged spheres becomes independent of simulation parameters for random sphere packings. For spherocylinder packings the contact number rises sharply at a critical volume fraction up to a value between 9 and 10 at the jamming point in line with the caging number $\langle\gamma\rangle=9$, the isostatic value $\langle C\rangle=10$ and the experimental value $\langle C\rangle=9.8 \pm 0.3$. The percentage of non-caged spherocylinders follows the same trend as the contact number as expected. The amount of order does not increase substantially with volume fraction while keeping the aspect ratio constant. At higher aspect ratio the contact number drops, which is probably due to the packing being not totally jammed.

The caging number for rods arrested by uncorrelated point contacts asymptotes towards $\langle\gamma\rangle=9$ at high aspect ratio according to the LCP prediction. This value is strikingly close to the experimental contact number $\langle C\rangle=9.8$ for thin rods, which confirms that thin-rod packings are dominated by local effects in the form of truly random neighbor cages. The aspect-ratio independence of the thin-rod caging number 
further validates the random contact equation. Together with a value for the contact number, either from an isostatic argument or a caging analysis, this law quantitatively explains the density of a random thin-rod packing.

Acknowledgments We are grateful to Stephen Williams for valuable discussions and Thijs Vlugt for his great help with setting up computational facilities. This work is part of the Granular Matter programme of the Stichting voor Fundamenteel Onderzoek der Materie (FOM), financially supported by the Nederlandse Organisatie voor Wetenschappelijk Onderzoek (NWO).

Open Access This article is distributed under the terms of the Creative Commons Attribution Noncommercial License which permits any noncommercial use, distribution, and reproduction in any medium, provided the original author(s) and source are credited.

\section{References}

1. Milewski, J.V.: Fiber-filled plastics. Ind. Eng. Chem. Prod. Res. Dev. 17, 363 (1978)

2. Nardin, M., Papirer, E., Schultz, J.: Contribution a l'etude des empilements au hasard de fibres et/ou de particules spheriques. Powder Technol. 44, 131 (1985)

3. Rahli, O., Tadrist, L., Blanc, R.: Experimental analysis of the porosity of randomly packed rigid fibers. C. R. Acad. Sci. Paris 327, 725 (1999)

4. Rudge, J.F., Holness, M.B., Smith, G.C.: Quantitative textural analysis of packings of elongate crystals. Contrib. Mineral. Petrol. 156, 413 (2008)

5. Philipse, A.P.: The random contact equation and its implications for (colloidal) rods in packings, suspensions, and anisotropic powders. Langmuir 12(5), 1127 (1996)

6. Philipse, A.P.: Corrigendum Langmuir 12, 5971 (1996)

7. Bernal, J.D.: A geometrical approach to the structure of liquids. Nature 183, 141 (1959)

8. Williams, S.R., Philipse, A.P.: Random packings of spheres and spherocylinders simulated by mechanical contraction. Phys. Rev. E 67, 051301 (2003)

9. Donev, A., Cisse, I., Sachs, D., Variano, E., Stillinger, F.H., Connelly, R., Torquato, S., Chaikin, P.M.: Improving the density of jammed disordered packings using ellipsoids. Science 303(5660), 990 (2004)

10. Wouterse, A., Williams, S.R., Philipse, A.P.: Effect of particle shape on the density and microstructure of random packings. J. Phys. Cond. Matter 19, 406215 (2007)

11. Blouwolff, J., Fraden, S.: The coordination number of granular cylinders. Europhys. Lett. 76, 1095 (2006)

12. Stokely, K., Diacou, A., Franklin, S.V.: Two-dimensional packing in prolate granular materials. Phys. Rev. E 67, 051302 (2003)

13. Desmond, K., Franklin, S.V.: Jamming of three-dimensional prolate granular materials. Phys. Rev. E 73, 031306 (2006)

14. Lumay, G., Vandewalle, N.: Compaction of anisotropic granular materials: experiments and simulations. Phys. Rev. E 70, 051314 (2004)

15. Lumay, G., Vandewalle, N.: Experimental study of the compaction dynamics for two-dimensional anisotropic granular materials. Phys. Rev. E 74, 021301 (2006)
16. Ramioli, M., Pournin, L., Liebling, T.M.: Vertical ordering of rods under vertical vibration. Phys. Rev. E 76, 021304 (2007)

17. Mustoe, G.G.W., Miyata, M.: Material flow analyses of non-circular shaped granular media using discrete element methods. J. Eng. Mech. 127, 1017 (2001)

18. Donev, A., Torquato, S., Stillinger, F.H.: Neighbor list collision -driven molecular dynamics simulation for nonspherical particles. i. algorithmic details. J. Comp. Phys. 202(2), 737 (2005)

19. Donev, A., Torquato, S., Stillinger, F.H.: Neighbor list collision-driven molecular dynamics simulation for nonspherical particles. ii. applications to ellipses and ellipsoids. J. Comp. Phys. 202(2), 765 (2005)

20. Peters, E.A.J.F., Kollmann, M., Barenbrug, Th.M.A.O.M., Philipse, A.P.: Caging of a d-dimensional sphere and its relevance for the random dense sphere packing. Phys. Rev. E 63, 021404 (2001)

21. Wouterse, A., Plapp, M., Philipse, A.P.: On the caging number of two-and three-dimensional hard spheres. J. Chem. Phys. 123(5), 054507 (2005)

22. Philipse, A.P., Verberkmoes, A.: Statistical geometry of caging effects in random thin-rod structures. Physica A 235, 186 (1997)

23. Silbert, L.E., Ertas, D., Grest, G.S., Halsey, T.C., Levine, D.: Geometry of frictionless and frictional sphere packings. Phys. Rev. E 65(3), 031304 (2002)

24. Pournin, L., Weber, M., Tsukahara, M., Ferrez, J.A., Ramaioli, M., Liebling, T.M.: Three-dimensional distinct element simulation of spherocylinder crystallization. Granul. Matter 7, 119 (2005)

25. Allen, M.P., Tildesley, D.J.: Computer Simulation of Liquids. Oxford Science Publications, Oxford (1987)

26. O'Hern, C.S., Silbert, L.E., Liu, A.J., Nagel, S.R.: Jamming at zero temperature and zero applied stress: the epitome of disorder. Phys. Rev. E 68, 011306 (2003)

27. $\mathrm{Xu}, \mathrm{N}$., Blawzdziewicz, J., O'Hern, C.S.: Reexamination of random close packing: ways to pack frictionless disks. Phys. Rev. E 71, 061306 (2005)

28. Onsager, L.: The effects of shape on the interaction of colloidal particles. Ann. N. Y. Acad. Sci. 51, 627 (1949)

29. Baraff, D.: Analytical methods for dynamic simulation of nonpenetrating rigid bodies. Comput. Graphics 23, 223 (1989)

30. Baraff, D.: Fast contact force computation for non-penetrating rigid bodies. In: Computer Graphics Proceedings (Siggraph), p. 23 (1994)

31. Eberly, D.H.: Game Physics. Morgan Kaufmann, Menlo Park (2004)

32. Wouterse, A., Philipse, A.P.: Geometrical cluster ensemble analysis of random sphere packings. J. Chem. Phys. 125, 194709 (2006)

33. Man, W.N., Donev, A., Stillinger, F.H., Sullivan, M.T., Russel, W.B., Heeger, D., Inati, S., Torquato, S., Chaikin, P.M.: Experiments on random packings of ellipsoids. Phys. Rev. Lett. 94(19), 198001 (2005)

34. Donev, A., Stillinger, F.H., Chaikin, P.M., Torquato, S.: Unusually dense crystal packings of ellipsoids. Phys. Rev. Lett. 92(25), 255506 (2004)

35. Chaikin, P.M., Donev, A., Man, W.N., Stillinger, F.H., Torquato, S.: Some observations on the random packing of hard ellipsoids. Ind. Eng. Chem. Res. 45, 6960 (2006)

36. Mansfield, M.L., Rakesh, L., Tomalia, D.A.: The random parking of spheres on spheres. J. Chem. Phys. 105, 3245 (1996)

37. Rodney, D., Fivel, M., Dendievel, R.: Discrete modeling of the mechanics of entangled materials. Phys. Rev. Lett. 95, 108004 (2005) 